\title{
Cause Analysis and Removal of Boundary Artifacts in Image Deconvolution
}

\author{
Ji-Yeon Lee ${ }^{\dagger}$, Nam-Yong Lee ${ }^{\dagger+}$
}

\section{ABSTRACT}

In this paper, we conducted a cause analysis on boundary artifacts in image deconvolution. Results of the cause analysis show that boundary artifacts are caused not only by a misuse of boundary conditions but also by no use of the normalized backprojection. Results also showed that the correct use of boundary conditions does not necessarily remove boundary artifacts. Based on these observations, we suggest not to use any specific boundary conditions and to use the normalized backprojector for boundary artifact-free image deconvolution.

Key words: Landweber, Richardson-Lucy, Conjugate Gradient Method, Free Boundary Condition, Normalized Backprojector

\section{INTRODUCTION}

The image deconvolution is a basic problem in image processing and has many applications. Examples include restoration of astronomical images, microscopy, and medical imaging, etc [1]. Many methods have been developed for the image deconvolution. For example, we have the classical Wiener filtering [2], Bayesian based approaches [3-5], wavelet deblurring [6,7], variational methods $[8,9,10]$, etc.

The image deconvolution, however, often produces undesirable artifacts in deconvolved images. The paper [11] classifies obstacles of the image deconvolution into following four categories; noise, insufficient deconvolution, boundary artifacts, and incorrect blurring model. In this paper our main concern is the removal of boundary artifacts.

The boundary artifacts in image deconvolution problem are caused by various factors, depending on deconvolution methods. For instance, classical

※ Corresponding Author: Lee Nam-Yong, Address: (621-749) 197 Inje-ro Kinhae Kyeong-Nam Kinhae Korea, TEL : +82-55-320-3860, FAX : +82-55-320-3254, E-mail : nylee@inje.ac.kr

Receipt date: Mar. 18, 2014, Revision date: May. 8, 2014 frequency domain filtering methods, which utilize the decoupling property of the circular convolution via fast Fourier transform (FFT), severely suffers from boundary artifacts, due to the mismatch in blurring models; the frequency domain filtering methods assumes a circular convolution as the blurring model, instead of a truncated convolution, which fits more accurately blurring models in many applications.

To remove boundary artifacts, various boundary condition methods have been proposed along with iterative deconvolution methods. The boundary condition (BC) method imposes certain condition on pixels across boundaries. Among them, periodic, reflective, and anti-reflective $\mathrm{BCs}$ have been most popular [12-15]. BC methods, however, often do not remove boundary artifacts effectively in case when imposed conditions are greatly mismatched to characteristic of images to be recovered.

In this paper, to investigate what really causes boundary artifacts, we conducted various simu-

\footnotetext{
Approval date: Jun. 26, 2014

+ Department of Computer Aided Science, Inje University (E-mail: jylee2930@gmail.com)

${ }^{++}$Department of Applied Mathematics, Inje University
} 
lation studies. Through a careful analysis on source of boundary artifacts, we suggest not to impose any specific $\mathrm{BCs}$ to avoid imposing mismatched $\mathrm{BCs}$ to characteristics of images to be restored. This result was preluded in the work of Calvetti et. al. [16]. The main result of the cause analysis is that no use of $\mathrm{BC}$ is not sufficient, while the use of the normalized backprojector which is designed to compensate for the non-uniformity in contributions from image pixels to the observation along with no use of $\mathrm{BC}$ removes boundary artifacts effectively. Simulation results in this paper will support this claim

\section{BACKGROUNDS}

\subsection{Problem of Image Deblurring}

The image deconvolution problem is to find the true image $f$ from the observed image $g$ which is blurred and noised as

$$
g=P f+n,
$$

where $P$ is the linear transform that determines the blurring process and $n$ represents a mean zero Gaussian noise. In this paper, we assume that the blurring by $P$ is space-invariant so that there $\mathrm{ex}^{-}$ ists a point spread function (PSF) $k$ such that $P f=k * f$ the convolution of $k$ and $f$. The exact definition of $k * f$ will be given in the next section.

In (1), to include every pixel of $f$ that affects the observed image $g$ through blurring, it is natural to assume that the domain $\Omega$ of the true image $f$ is larger than the domain $\Lambda$ of the observed image $g$. The blurring process makes some near-boundary pixels of the observed image $g$ to be influenced by 'unseen pixels' (pixels in $\Omega-\Lambda$ ). It is often regarded that the existence of unseen pixels causes boundary artifacts [12].

In this paper, we will use following notations and terminologies.

- $g=P f+n$ : the observation model, where $g$ is the observed image, $f$ is the true image to be re $^{-}$ stored, $P$ is the projector that represents the space-invariant blurring, and $n$ is a mean zero Gaussian noise.

- $f=\left(f_{j_{1}, j_{2}}\right)$ : the image, $f$, will be denoted in an alphabet, while its image pixel value, $f_{j_{1}, j_{2}}$, at $\left(j_{1}, j_{2}\right)$, will be denoted in the same alphabet with subscripted indices. With the single pixel index., we will treat image $g$ and $f$ as one dimensional vectors. The same rule will hold for other images and PSFs throughout this paper.

- $\Lambda$ : the set of image pixels where $g$ is defined.

- $\Omega$ : the set of image pixels where $f$ is defined.

- $\Omega-\Lambda$ : the set of "unseen image pixels".

- $L^{2}(\Omega)$ : the set of images defined on $\Omega$.

- $L^{2}(\Lambda)$ : the set of images defined on $\Lambda$.

- $k$ : the PSF that defines the blurring transform $P$ by

$$
(P f)_{b}=\sum_{v \in \Omega} P_{b, v} f_{v}=\sum_{v \in\left(b-S_{k}\right)} k_{b-v} f_{v},
$$

where $S_{k}=\left\{v \mid k_{v} \neq 0\right\}$. The blurring transform $P$ will be called projector throughout this paper.

Throughout this paper, we assume that the PSF $k$ satisfies following conditions:

$$
k_{i_{1}, i_{2}} \geq 0, \quad \sum_{i_{1}, i_{2} \in S_{k}} k_{i_{1}, i_{2}}=1,(0,0) \in S_{k}
$$

\subsection{Iterative Methods}

\section{Landweber Method (LM)}

For $g=P f$, the Landweber iteration takes

$f_{n+1}=f_{n}+\beta P^{t}\left(g-P f^{n}\right)$

Here, $\beta$ the step length must have the value of

$$
0<\beta<\frac{2}{\sigma_{\max }^{2}},
$$

where $\sigma_{\max }$ is the largest singular value of $P$. For details and applications of Landweber method, see, e.g., [17].

\section{Richardson-Lucy (RL)}

For $g=P f$, starting from $f^{0}=1_{\Omega}$, where $1_{\Omega}$ is the all-one image defined on $\Omega$, the Richardson-Lucy 
method takes

$$
f^{n+1}=f^{n} \cdot{ }^{*} P^{t} s^{n} \cdot / P^{t} 1_{\Lambda}, \quad s^{n}=g \cdot / P f^{n},
$$

where, .* and ./ are pixel-by-pixel multiplication and division between two images. and $1_{\Lambda}$ is the all-one images defined on $\Lambda$. For details and applications of Richardson-Lucy method, see, e.g., $[4,5]$.

\section{Conjugate Gradient Least Square (CGLS)}

The conjugate gradient method is designed for the linear system governed by positive definite matrix [18]. For (1), we consider the Tikhonov regularization method formulated by

$$
\min _{f}\left(\|g-P f\|_{2}+\lambda\|f\|_{2}^{2}\right),
$$

where $\lambda>0$ is a regularization parameter and $\|\cdot\|_{2}$ is the $L^{2}-$ norm. This variational problem leads to the following normal equation:

$$
\left(P^{t} P+\lambda I_{\Omega}\right) f=P^{t} g .
$$

Since the governing matrix $P^{t} P+\lambda 1_{\Omega}$ in (5) is positive definite, the conjugate gradient method is applicable to (5). We will denote this method by Conjugate Gradient Least Square (CGLS).

\subsection{Boundary Conditions}

In this section, we will explain various $\mathrm{BCs}$ such as periodic, reflective, and anti-reflective boundary conditions.

To simplify the presentation, we assume that

$$
\Lambda=\left\{\left(i_{1}, i_{2}\right) \mid 0 \leq i_{\nu}<N_{\nu}\right\}, \nu=1,2
$$

for some positive integers $N_{1}, N_{2}$, and

$$
S_{k}=\left\{\left(i_{1}, i_{2}\right) \mid-L_{\nu} \leq i_{\nu} \leq M_{\nu}\right\}, \nu=1,2
$$

for some positive integers $L_{1}, L_{2}, M_{1}, M_{2}$. In this case,

$$
\Omega=\left\{\left(j_{1}, j_{2}\right) \mid-M_{\nu} \leq j_{\nu} \leq N_{\nu}+L_{v}-1\right\},
$$

where $\nu=1,2$

We divide the true image $f$ into 9 parts as follows

$$
f=\left[\begin{array}{lll}
f_{n w} & f_{n} & f_{n e} \\
f_{w} & f_{c} & f_{e} \\
f_{s w} & f_{s} & f_{s e}
\end{array}\right],
$$

where $f_{c}=\left(f_{i_{1}}, f_{i_{2}}\right), \quad 0 \leq i_{\nu}<N_{\nu} \quad(\nu=1,2) \quad$ repre $^{-}$ sents the image part defined on $\Lambda$; the others represent the image part defined on $\Omega-\Lambda$, the set of unseen image pixels across the boundary of $\Lambda$. Boundary condition method impose certain restriction on $f_{n w}, f_{n}, f_{n e}, f_{w}, f_{e}, f_{s w}, f_{s}$ and $f_{s e}$ in (9).

\section{Periodic BC (PBC)}

In 2-Dimensional, the $i$-th row of the periodic boundary condition imposed images is

$$
\begin{array}{r}
\left(f_{i, N_{2}-M_{2}} \cdots, f_{i, N_{2}-2}, f_{i, N_{2}-1},\right. \\
f_{i, 0}, f_{i, 1}, \cdots, f_{i, N_{2}-2_{2}}, f_{i, N_{2}-1}, \\
\left.f_{i, 0}, f_{i, 1}, \cdots, f_{i, L_{2}-1}\right)
\end{array}
$$

and similarly for the column.

\section{Reflective BC (RBC)}

The reflective boundary condition, in 2-dimensional, imposed image is as follows

$$
\begin{aligned}
& \left(f_{i, M_{2}-1}, \cdots, f_{i, 1}, f_{i, 0},\right. \\
& \quad f_{i, 0}, f_{i, 1}, \cdots, f_{i, N_{2}-2_{2}}, f_{i, N_{2}-1}, \\
& \left.\quad f_{i, N_{2}-1}, f_{i, N_{2}-2} \cdots, f_{i, N_{2}-L_{2}}\right)
\end{aligned}
$$

and similarly for the column.

\section{Anti-Reflective BC (ABC)}

The anti-reflective boundary condition, in $2-\mathrm{di}^{-}$ mensional, imposed image is as follows

$$
\begin{aligned}
& \left(2 f_{i, 0}-f_{i, M_{2}}, \cdots, 2 f_{i, 0}-f_{i, 2}, 2 f_{i, 0}-f_{i, 1},\right. \\
& \quad f_{i, 0}, f_{i, 1}, \cdots, f_{i, N_{2}-2}, f_{i, N_{2}-1}, \\
& \left.\quad 2 f_{i, N_{2}-1}-f_{i, N_{2}-2}, \cdots, 2 f_{i, N_{2}-1}-f_{i, N_{2}-L_{2}-1}\right)
\end{aligned}
$$

and similarly for the column.

Any set of BCs introduce an extension operator $E: L^{2}(\Lambda) \rightarrow L^{2}(\Omega)$ such that

$$
E f_{c}=\left[\begin{array}{ccc}
f_{n w}^{*} & f_{n}^{*} & f_{n e}^{*} \\
f_{w}^{*} & f_{c}^{*} & f_{e}^{*} \\
f_{s w}^{*} & f_{s}^{*} & f_{s e}^{*}
\end{array}\right]
$$


where $f_{n w}^{*}, f_{n}^{*}, f_{n e}^{*}, f_{w}^{*}, f_{e}^{*}, f_{s w}^{*}, f_{s}^{*}$, and $f_{s e}^{*}$ represent parts of the image imposed by boundary condition. By using extension operator $E$ associated with $\mathrm{PBC}, \mathrm{RBC}$, and $\mathrm{ABC}$ can be defined by (10), (11), and (12), respectively. $\mathrm{BC}$ methods use the $\mathrm{ex}^{-}$ tension operator $E$ to modify (1) to

$$
g=P E f_{c}+n=\tilde{P} f+n .
$$

Iterative deblurring methods described in section 2.3 can be applied to (14) by replacing $f$ with $f_{c}$ and $P$ with $P E$.

One of the main advantage of $\mathrm{BC}$ methods is the computational efficiency. For example, PBC, RBC, and $\mathrm{ABC}$ based models can be diagonalizable by FFT, discrete cosine transform (DCT), and sine transform (DST), respectively. For details, see. e.g., [13-15].

\section{Free BC (FBC)}

Instead of imposing specific $\mathrm{BCs}$, works in $[15,18,19]$ suggest not to use them. In [19], Calvetti et. al. claim that "In an Aristotelian approach to knowledge, when it is not known a priori which BCs should be chosen, by admitting our lack of information it is possible to let the data itself $\mathrm{de}^{-}$ termine them." In [19], this no use of $\mathrm{BC}$ approach is called free $\mathrm{BC}(\mathrm{FBC})$. Thus, $\mathrm{FBC}$ does not modify the deconvolution problem (1).

We will use the notation, 'iterative method'' $\mathrm{BC}$ ', to describe the iterative deblurring method and the boundary condition. For example, CGLS$\mathrm{RBC}$ and CGLS-ABC are CGLS iteration with reflective $\mathrm{BC}$ and anti-reflective $\mathrm{BC}$, respectively.

\section{PROPOSED METHOD}

\subsection{Cause Analysis}

To investigate the source of boundary artifacts, we conducted simulations with test images in Fig. 1. Fig. 1 shows images satisfying various $\mathrm{BCs}$ ((a) $\mathrm{PBC}$, (b) RBC, (c) $\mathrm{ABC}$, (d) no $\mathrm{BC}$ ) on 10 pixel rows on the upper boundary and 10 pixel columns
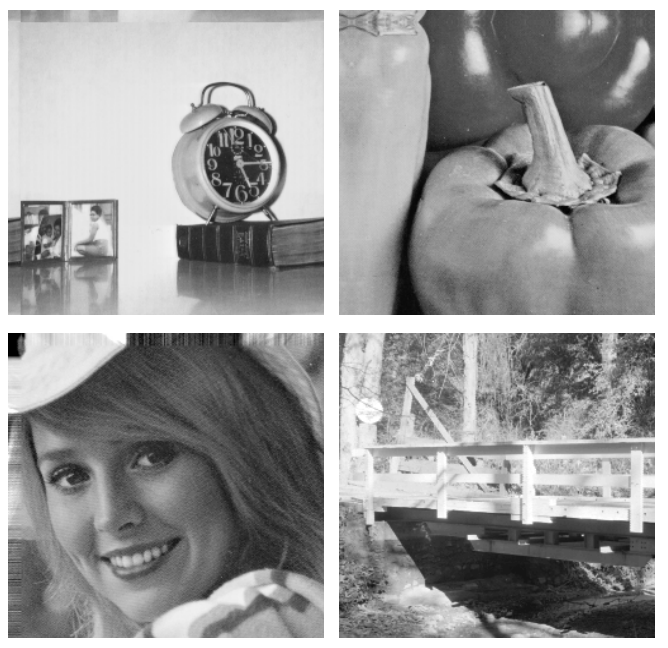

Fig. 1. Test images. (a) (upper-left) 'clock' satisfying PBC, (b) (upper-right) 'peppers' satisfying RBC, (c) (lower-left) 'girl' satisfying ABC, (d) (lowerright) 'bridge' satisfying no BCs. All images are of size $250 \times 250$.

on the left boundary. In this paper, we used $11 \times 11$ diagonal PSF $k_{D}$ whose diagonal elements are $\frac{1}{275}\{30,29, \ldots, 20\}$ and the first diagonal element $\frac{30}{275}$ is $k_{0,0}$. Notice that the location of $\mathrm{BC}$-imposed image pixels in Fig. 1 is determined by the support of the PSF $k_{D}$.

Fig. 2 shows blurred versions of images in Fig.
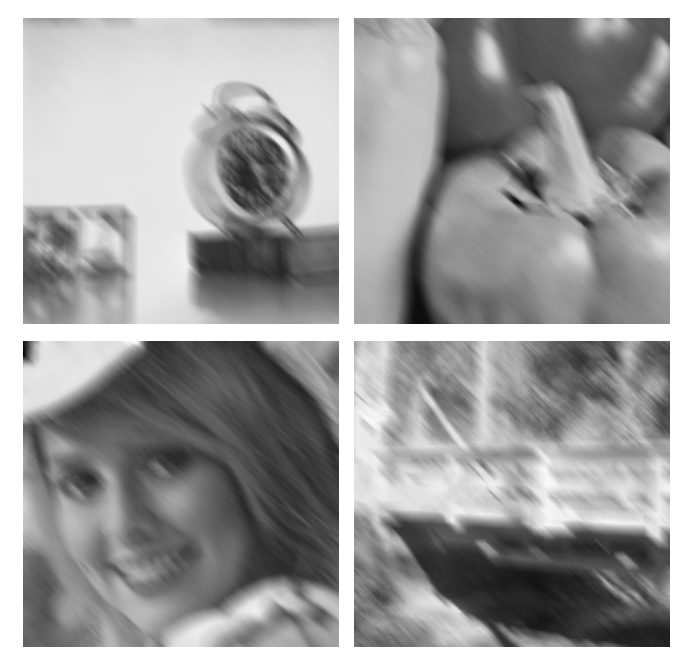

Fig. 2. Blurred images by the $11 \times 11$ diagonal PSF $k_{D}$ from images in Fig. 1. All blurred images are of size $240 \times 240$. 
1 by the $11 \times 11$ diagonal PSF $k_{D}$. In this simulation, we did not add noise to exclude noise-related artifacts in deconvolved images for a better visual comparison on boundary artifact removal.

Fig. 3 shows deconvolved images by LM from images in Fig. 2. In Fig. 3, we presented restored images on $\Lambda$, if necessary (Fig. 3(d)), by cropping out image pixels on $\Omega-\Lambda$. Here we note that LM-PBC (Fig. 3(a)), LM-RBC (Fig. 3(b)), and LM-ABC (Fig. 3(c)) recover images $f_{c}$ defined on $\Lambda$, while LM-FBC recovers $f$ defined on $\Omega$. In Fig. 3(c)(lower-left) satisfying ABC, the boundary artifacts were very severe even though the image was obtained by only 10 iterations. Other images in Fig. 3 were obtained by 1000 iterations. In this simulation, we used a fixed parameter $\beta=0.25$ which is the step length of LM iterations in (6) for all pixels. Smaller step lengths $\beta$ can reduce the artifacts in Fig. 3(c), but, at the same time, it decreases the convergence rate.

In the simulation for Fig. 3, original images to be recovered are assumed to satisfy imposed BCs, as seen in Fig. 1. Thus, in the simulation for Fig. 3 , there were no unseen pixels across upper and left boundaries in observed images in Fig. 2. Some
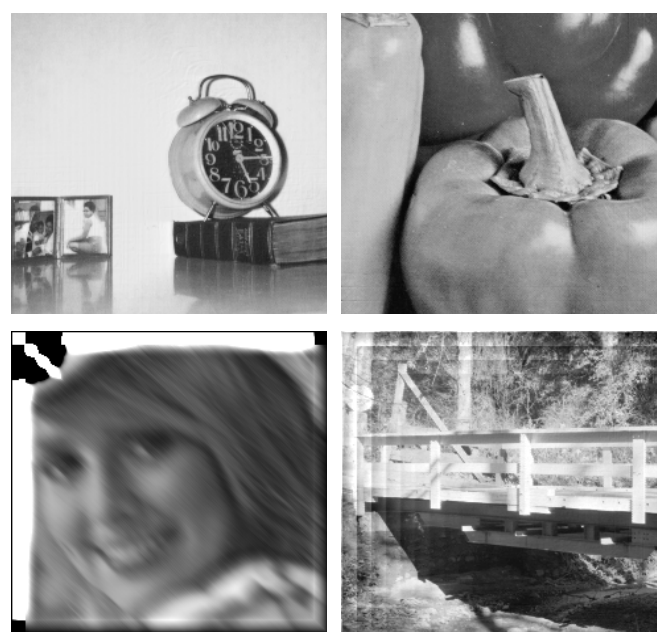

Fig. 3. Deconvolved images by LM from blurred images in Fig. 2 by using (a) (upper-left) PBC, (b) (upper-right) RBC, (c) (lower-left) ABC, and (d) (lower-right) FBC. of restored images ((c), (d)) in Fig. 3, however, exhibited boundary artifacts. These simulation $\mathrm{re}^{-}$ sults show that correctly imposed $\mathrm{BC}$ does not guarantee boundary artifact removal in LM.

Fig. 4 shows deconvolved images by RL from images in Fig. 2. Again, in Fig. 3, we presented restored images only on $\Lambda$.

In the simulation for Fig. 4, original images to be recovered are also assumed to satisfy imposed BCs, as in the simulation for Fig. 3. Thus, again, in the simulation for Fig. 4, there were no unseen pixels across upper and left boundaries in observed images in Fig. 2. Fig. 4(c), however, exhibited severe boundary artifacts.

Restored images by RL showed different implications for ABC (Fig. 4(c)) as compared with PBC (Fig. 4(a)), RBC (Fig. 4(b)), and FBC (Fig. 4(d)). Boundary artifacts in RL-ABC (Fig. 4(c)) can be explained by following argument. Notice that $\mathrm{RL}-\mathrm{ABC}$ takes the iteration

$$
f_{\Lambda}^{n+1}=f_{\Lambda}^{n} \cdot * E^{t} P^{t} s^{n} \cdot / E^{t} P^{t} 1_{\Lambda}, \quad s^{n}=g \cdot / P E f_{\Lambda}^{n},
$$

where $E$ is the extension transform associated with $\mathrm{ABC}$. It is well known that RL-type iterations does not provide accurate estimation for pixels $v$
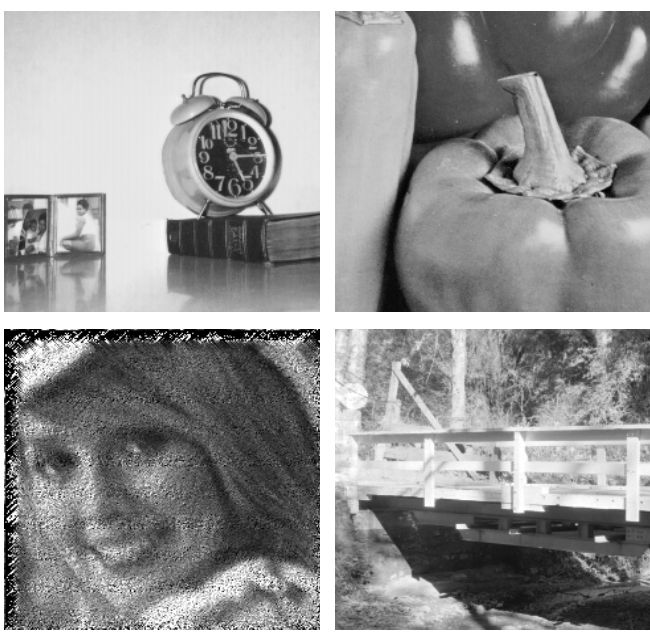

Fig. 4. Deconvolved images by RL from blurred images in Fig. 2 by using (a) (upper-left) PBC, (b) (upper-right) RBC, (c) (lower-left) ABC, and (d) (lower-right) FBC. 
where the denominator $\left(E^{t} P^{t} 1_{\Lambda}\right)_{v}=0$. The characteristic of the extension transform $E$ associated with $\mathrm{ABC}$, however, makes $\left(E^{t} P^{t} 1_{\Lambda}\right)_{v}=0$ for some pixel $v$. This result shows that $\mathrm{RL}-\mathrm{ABC}$ is not a good approach for the image deconvolution.

As compared with LM-FBC (Fig. 3(d)), RLFBC (Fig. 4(d)) did not show boundary artifacts. Since FBC means no use of BCs, this result show that the boundary artifact removal can be archived without using correct $\mathrm{BCs}$.

As seen in Fig. 3(d), FBC alone, however, does not remove boundary artifacts [20]. Despite the failure of $\mathrm{FBC}$ in removing boundary artifacts, we suggest FBC as the first step to avoid boundary artifacts caused by the use of mismatched BCs, by noting that boundary artifacts in FBC can be easily removable by using the normalized backprojector. The exact definition of the normalized backprojector and detailed explanation will be given in the next section.

\subsection{Normalized Backprojector}

Simulation results in Section 3.1 show that the use of correct $\mathrm{BC}$ does not guarantee boundary artifact removal in LM and RL. Moreover, the result in Fig. 4(d) by RL-FBC shows that boundary artifacts can be removed without using correct BCs. The comparison of Fig. 3(d) by LM-FBC with Fig. 4(d) by RL-FBC indicates that the boundary artifact can be removed not by imposing correct $\mathrm{BC}$ but by using special characteristic of RL that is not in LM.

To find out what special characteristic of RL leads to boundary artifact removal, we reformulate the Landweber method (4) in the following generalized form [21]:

$$
f_{n+1}=f_{n}+B_{n} P^{t} M_{n}\left(g-P f_{n}\right),
$$

where $B_{n}=\operatorname{diag}\left\{\beta_{1}^{(n)}, \beta_{2}^{(n)}, \cdots, \beta_{\Omega}^{(n)}\right\}$ with positive elements controlling step size of each iteration and positive definite matrices $M_{n}$. Notice that $\mathrm{RL}$ also can be written in an addition form (16) with

$$
B_{n}=f_{n} \cdot / P^{t} 1_{\Lambda} \text { and } M_{n}=\operatorname{diag}\left\{1_{\Lambda} \cdot / P f_{n}\right\}
$$

This shows that the main difference between $\mathrm{LM}$ and $\mathrm{RL}$ is the use of the normalized term $P^{t} 1_{\Lambda}$ in the denominator. We call $1_{\Omega} \cdot / P^{t} 1_{\Lambda} \cdot{ }^{*} P^{t}$ normalized backprojector (NBP) by noting that it backprojects the all-one image $1_{\Lambda}$ on $\Lambda$ to the all-one image $1_{\Omega}$ on $\Omega$.

We denote the modified LM that uses the normalized backprojector $1_{\Omega} \cdot / P^{t} 1_{\Lambda} \cdot{ }^{*} P^{t}$ instead of the standard backprojector $P^{t}$ with $\mathrm{FBC}$ by $\mathrm{LM}^{-}$ NFBC. We also use LM-NPBC, LM-NRBC, LM$\mathrm{NABC}$ to refer $\mathrm{NBP}-$ based $\mathrm{LM}$ with $\mathrm{PBC}, \mathrm{RBC}$, and $\mathrm{ABC}$, respectively. With the same argument, we define CGLS-NFBC. Here we note that RLNPBC, RL-NRBC, RL-NABC, RL-NFBC are same as RL-PBC, RL-RBC, RL-ABC, RL-FBC, respectively.

To check whether the normalized backprojector $1_{\Omega} \cdot / P^{t} 1_{\Lambda} \cdot{ }^{*} P^{t}$ can remove boundary artifacts in LM, we conducted image deconvolution simulation by NBP based LM.

Fig. 5 shows deconvolved images by NBP based LM from images in Fig. 2. As in simulations for
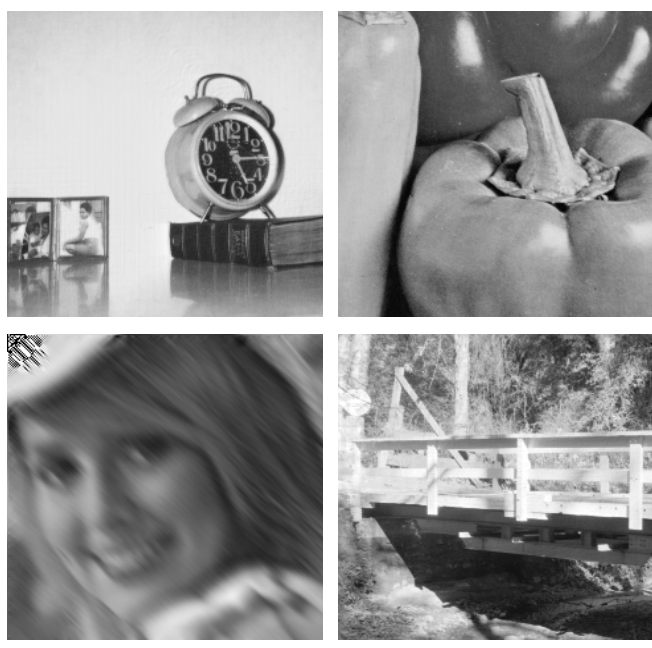

Fig. 5. Deconvolved images from blurred images in Fig. 2 by using (a) (upper-left) LM-NPBC, (b) (upper-right) LM-NRBC, (c) (lower-left) LM$\mathrm{NABC}$, and (d) (lower-right) LM-NFBC. 
Fig. 3 and 4, in the simulation for Fig. 5, original images to be recovered are assumed to satisfy imposed BCs, as seen in Fig. 1, and hence there were no unseen pixels across upper and left boundaries in observed images in Fig. 2.

Fig. 5(a) by LM-NPBC was exactly identical to Fig. 3(a) by LM-PBC. This phenomenon can be explained by the following argument. Notice that the standard backprojector $(P E)^{t}$ of $\mathrm{LM}-\mathrm{PBC}$, where $E$ is the extension transform associated with $\mathrm{PBC}$, is already normalized, i.e., $(P E)^{t} 1_{\Lambda}=1_{\Omega}$ be $^{-}$ cause of conditions (3) imposed on the PSF $k$.

Fig. 5(b) by LM-NRBC showed a slight improvement over Fig. 3(b) by LM-RBC.

Fig. 5(c) by LM-NABC exhibited severe boundary artifacts as in Fig. 4(c) by RL-ABC. Boundary artifacts in LM-NABC (Fig. 5(c)) can be $\mathrm{ex}^{-}$ plained by the same argument used for Fig. 4(c) by $\mathrm{RL}-\mathrm{ABC}$, since both methods use $E^{t} P^{t} 1_{\Lambda}$ (here $E$ is the extension operator associated with $\mathrm{ABC}$. Notice that this term can be 0 for some pixels) as the normalized term in their backprojectors. This result shows that $\mathrm{ABC}$ is not good for NBP.

Fig. 5(d) by LM-NFBC showed a clear improvement over Fig. 3(d) by LM-FBC. All boundary artifacts were removed. Based on these observations, we suggest not to use any specific BCs and to use the normalized backprojector for boundary artifact removal. In other words, we suggest to use iterative methods based on NFBC.

\subsection{Implementation}

To explain how FBC is implemented, we will use commands of Matlab [1]. Before we do this, let us recall that if $\Omega=R^{M \times M}, \Lambda=R^{N \times N}$, and $k$ is a $L \times L$ sized PSF, then $M=N+L-1$.

As mentioned earlier, $\mathrm{FBC}$ does not use an $\mathrm{ex}^{-}$ tension operator $E$. Thus, the implementation of FBC depends on how the projection (the multiplication of the matrix $P$ and images on $\left.L^{2}(\Omega)\right)$ and the backprojection (the multiplication of the trans- pose matrix $P^{t}$ and images on $\left.L^{2}(\Lambda)\right)$ are computed. For these computations, we first compute optical transfer functions $K$ and $K^{(r)}$ of the PSF $k$ and its reversely ordered PSF $k^{(r)}$ (i.e., $k_{i_{1}, i_{2}}^{(r)}=k_{-i_{1},-i_{2}}$ ) by using following commands:

$$
\begin{aligned}
& >>Z=Z^{(r)}=\operatorname{zeros}(M, M) ; \\
& >>(1: L, 1: L)=k ; \\
& >>Z^{(r)}(1: L, 1: L)=k^{(r)} ; \\
& >>K^{\prime r} \operatorname{fft} 2(Z) ; \\
& >>K^{(r)}=\operatorname{fft} 2\left(Z^{(r)}\right) ;
\end{aligned}
$$

With these optical transfer functions $K$ and $K^{(r)}$, we can compute $y=P f$ and $x=P^{t} g$ respectively by

$>Y=\operatorname{real}(\operatorname{ifft} 2(K * \mathrm{fft} 2(f)))$;

$>y=Y(L: M, L: M)$;

(taking-out)

and

$>\quad G=\operatorname{zeros}(M, M)$;

$>G(1: N, 1: N)=g$

(zero-padding)

$>\quad x=\operatorname{real}\left(\mathrm{ifft} 2\left(K^{(r)} * \mathrm{fft}_{2}(G)\right)\right)$;

In case when $\mathrm{PBC}$ is used, we uses the extension operator $E$ to change the problem $g=P f+n$ (1) to $g=\tilde{P} f_{c}+n$ (14), where $\tilde{P}=P E$. For computations of projection and backprojection associated with $\mathrm{PBC}$, we first compute $\mathrm{PBC}$-related optical transfer functions $K_{p}$ and $K_{p}^{(r)}$ of $k$ and $k^{(r)}$, respectively, by using following commands:

$$
\begin{aligned}
& >>Z_{p}=Z_{p}^{(r)}=\operatorname{zeros}(N, N) ; \\
& >>Z_{p}(1: L, 1: L)=k ; \\
& >>Z_{p}^{(r)}(1: L, 1: L)=k^{(r)} ; \\
& >>K_{p}=\operatorname{fft} 2\left(Z_{p}\right) ; \\
& >>K_{p}^{(r)}=\operatorname{fft} 2\left(Z_{p}^{(r)}\right) ;
\end{aligned}
$$

Here we note that the main difference between $K, K^{(r)}$ (in $\mathrm{FBC)}$ ) and $K_{p}, K_{p}^{(r)}$ (in $\mathrm{PBC)}$ is the size of optical transfer function; $K$ and $K^{(r)}$ are of size $M \times M$, while $K_{p}$ and $K_{p}^{(r)}$ are of size $N \times N$.

With these optical transfer functions $K_{p}$ and $K_{p}^{(r)}$, we can compute $y=\tilde{P} f_{c}$ and $x=P^{t} g \mathrm{re}^{-}$ spectively by

$$
>y=\operatorname{real}\left(\operatorname{ifft} 2\left(K_{p} \cdot * \mathrm{fft} 2\left(f_{c}\right)\right)\right) ;
$$


and

$>x=\operatorname{real}\left(\operatorname{ifft} 2\left(K_{p}^{(r)} \cdot * \mathrm{fft} 2(g)\right)\right)$;

In case when $\mathrm{RBC}$ or $\mathrm{ABC}$ are used, we can have similar computational efficiencies to $\mathrm{PBC}-\mathrm{re}^{-}$ lated one by replacing ' $\mathrm{fft} 2$ ' and 'ifft2' with ' $\mathrm{dct} 2$ ' and 'idct2' for RBC and 'dst2' and 'idst2' for ABC, where 'dct' and ' $\mathrm{dst}^{\prime}$ represent DCT and DST, respectively.

The computational efficiency comparison between FBC and PBC clearly shows that FBC can be implemented with a fast algorithm, which requires only few extra computations for 'bigger sized optical transfer functions', 'taking-out' and 'zero-padding' than PBC based algorithms. Simulation results in this paper will show that those extra computations in FBC pay off by removing boundary artifacts more efficiently than $\mathrm{PBC}, \mathrm{RBC}$, and $\mathrm{ABC}$.

\section{SIMULATION STUDIES}

We conducted simulations to test the performance of the proposed method NFBC. Fig. 6 shows test images (a) 'moon' and (b) ‘boat'. Fig. 7 (a) and (b) show blurred and noised images by $11 \times 11$ Gaussian PSF $k$ and $11 \times 11$ diagonal PSF $k_{D}$, respectively. We added mean zero Gaussian noises to blurred images. Here standard deviations of added noises were set to be $0.5 \%$ of means of blurred images

In simulations, we chose the image that had the smallest relative square error (RSE) in 500 iterations as the deconvolved image by the tested
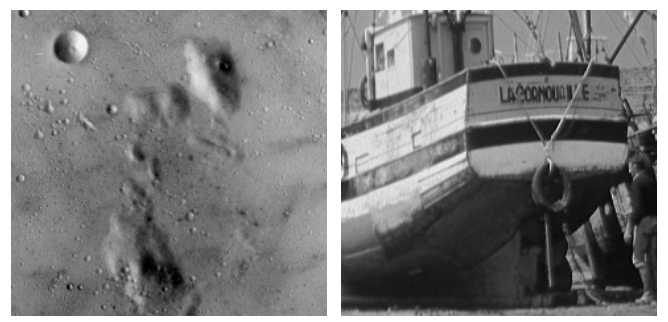

Fig. 6. Test images. (a) 'moon', (b) 'boat'. The size of test images is $250 \times 250$.
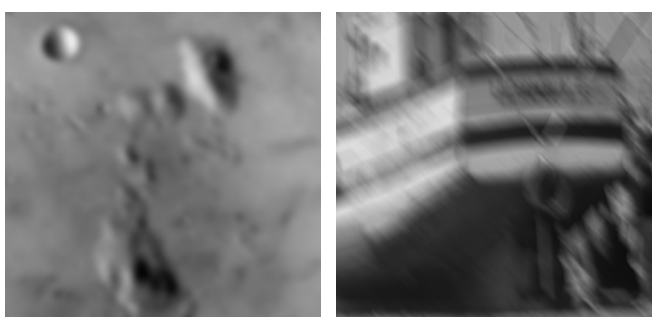

Fig. 7. Blurred, by (a) $k$ and (b) $k_{D}$, and noised images. The noise term was generated by mean zero Gaussian noise with the standard deviation = $0.5 \%$ of the mean of the blurred image. Here, we used $11 \times 11$ Gaussian PSF $k_{G}$ with $\sigma=2.5$ pixel unit length and $11 \times 11$ diagonal PSF $k_{D}$ which was explained in section 3.1.

method. Here the RSE is defined by

$$
\operatorname{RSE}=\frac{\sum_{\left(i_{1}, i_{2}\right) \in \Lambda}\left|\tilde{f}_{i_{1}, i_{2}}-f_{i_{1}, i_{2}}\right|^{2}}{\sum_{\left(i_{1}, i_{2}\right) \in \Lambda}\left|f_{i_{1}, i_{2}}\right|^{2}} \times 100(\%),
$$

where, $\tilde{f}_{i_{1}, i_{2}}$ and $f_{i_{1}, i_{2}}$ are pixel values of the deconvolved image and the true image, respectively.

Fig. 8 shows deconvoloved images by CGLSPBC. Results showed that boundary artifacts in the Gaussian deconvolution (Fig. 8(a)) and more severe ones in the diagonal deconvolution (Fig. 8(b)). The relative differences in boundary artifacts are caused by facts that the Gaussian PSF $k_{G}$ decays more rapidly than the diagonal PSF $k_{D}$ and the 'moon' image has boundaries which are relatively more matched to $\mathrm{PBC}$ than the 'boat' image.

CGLS-PBC attains the smallest RSE at 7 iter-
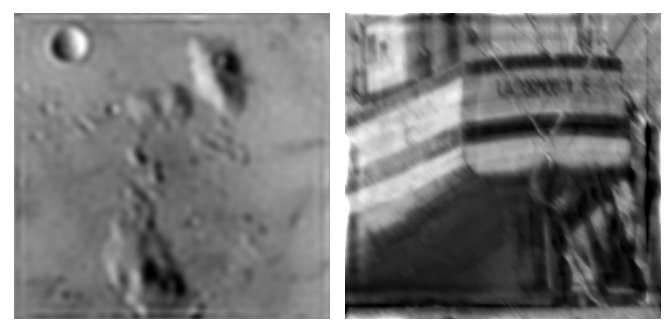

Fig. 8. Deconvolved images by CGLS-PBC from (a)(left) Fig. 7(a) and (b)(right) Fig. 7(b). The image in (a) was obtained at 7 iterations with RSE $=0.52 \%$ and the image in (b) was obtained at 5 iterations with RSE $=3.70 \%$. 
ations with $\mathrm{RSE}=0.52 \%$ for Fig. 8 (a) and at 5 iterations with RSE $=3.70 \%$ for Fig. 8(b). These results indicate that boundary artifacts caused by the mismatch of $\mathrm{PBC}$ to the image characteristic prevents CGLS-PBC from attaining smaller RSE with longer iterations.

Fig. 9 shows deconvoloved images by CGLSRBC. Results showed that severe boundary artifacts in the diagonal deconvolution in Fig. 9(b), while boundary artifacts in the Gaussian deconvolution in Fig. 9(a) were hardly noticeable. The comparison between Fig. 8(a) by CGLS-PBC and Fig. 9(a) by CGLS-RBC indicates that RBC is relatively more matched to the boundary characteristic of the 'moon' image than PBC. As seen in Fig. 9(b) by CGLS-RBC, the mismatch between the boundary characteristic of the 'boat' image and RBC caused severe boundary artifacts.

CGLS-RBC attains the smallest RSE at 29 iterations with RSE $=0.42 \%$ for Fig. $9(\mathrm{a})$ and at 17 iterations with $\mathrm{RSE}=1.47 \%$ for Fig. $8(\mathrm{~b})$. The comparison of these results with those in Fig. 8 also indicates that the mismatch of $\mathrm{RBC}$ is less severe than that of $\mathrm{PBC}$.

Fig. 10 shows deconvolved images by CGLSABC. Results showed that both the Gaussian deconvolution (a) and the diagonal deconvolution (b) suffer from boundary artifacts.

CGLS-ABC attains the smallest RSE at 486 iterations with RSE $=0.81 \%$ for Fig. 10(a) and at 124 iterations with RSE $=1.10 \%$ for Fig. 10(b).
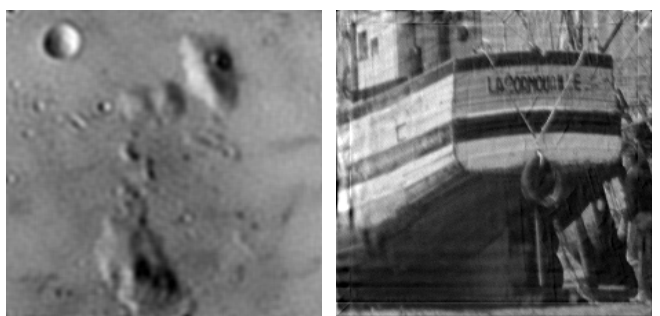

Fig. 9. Deconvolved images by CGLS-RBC from (a)(left) Fig. 7(a), and (b)(right) Fig. 7(b). The image in (a) was obtained at 29 iterations with RSE = $0.42 \%$ and the image in (b) was obtained at 17 iterations with $\mathrm{RSE}=1.47 \%$.
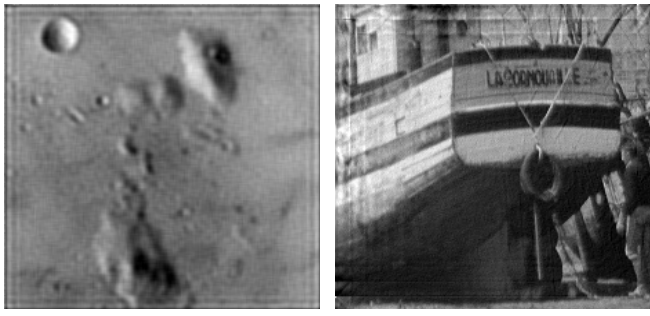

Fig. 10. Deconvolved images by CGLS-ABC from (a) (left) Fig. 7(a) and (b)(right) Fig. 7(b). The image in (a) was obtained at 486 iterations with RSE $=0.81 \%$ and the image in (b) was obtained at 124 iterations with RSE $=1.10 \%$.

The comparison of these results with those in Fig. 8 and 9 indicates that $\mathrm{ABC}$ decreases the convergence rate.

Fig. 11 shows deconvoloved images by CGLSFBC (the first row) and CGLS-NFBC (the second row). Results using FBC only showed that both the Gaussian deconvolution (Fig. 11(a)) and the diagonal deconvolution (Fig. 11(b)) suffer from boundary artifacts. CGLS-FBC attains the smallest
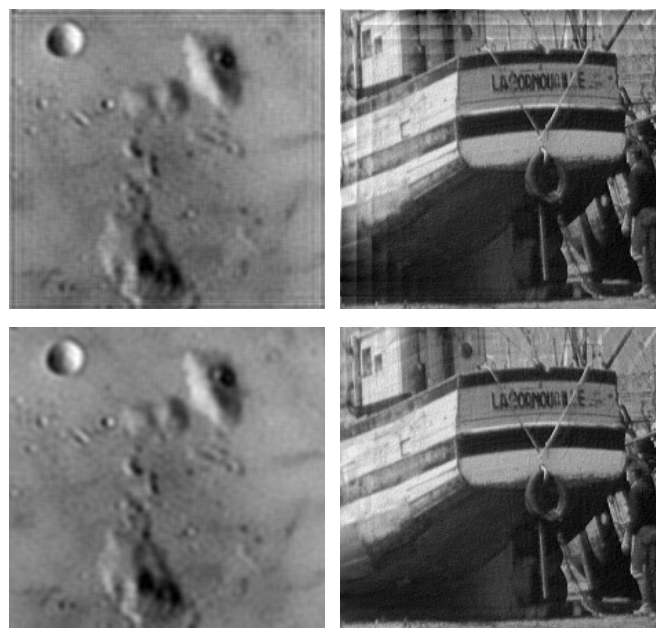

Fig. 11. Deconvolved images by CGLS-FBC and CGLSNFBC from (a and c) Fig. 7(a) and (b and d) Fig. 7(b). The image in (a)(upper-left) was obtained at 68 iterations with RSE $=0.56 \%$ by CGLS-FBC, the image in (b)(upper-right) was obtained at 56 iterations with RSE $=0.94 \%$ by CGLS-FBC, the image in (c)(lower-left) was obtained at 54 iterations with RSE $=0.41 \%$ by CGLS-NFBC, and the image in (d)(lower-right) was obtained at 61 iterations with RSE $=0.47 \%$ by CGLS-NFBC, 
RSE at 68 iterations with RSE $=0.56 \%$ for Fig. 11 (a) and at 56 iterations with $\mathrm{RSE}=0.94 \%$ for Fig. 11(b).

Fig. 11(c) and 11(d) show deconvoloved images by CGLS-NFBC. Boundary artifacts were effectively removed both in the Gaussian deconvolution (Fig. 11(c)) and the diagonal deconvolution (Fig. 11(d)). CGLS-NFBC attains the smallest RSE at 54 iterations with $\mathrm{RSE}=0.41 \%$ for Fig. $11(\mathrm{c})$ and at 61 iterations with $\mathrm{RSE}=0.47 \%$ for Fig. 11(d). Thus, CGLS-NFBC achieved the smallest RSE in this simulation.

These results show that the use of NFBC $\mathrm{re}^{-}$ moves the chance of using mismatched $\mathrm{BCs}$, as seen in Fig. 8(b) and 9(b), and efficiently removes boundary artifacts by using NBP. The comparison between Fig. 11(a) and 11(b) by FBC only and Fig. 11(c) and 11(d) by $\mathrm{FBC}+\mathrm{NBP}$ supports the latter claim.

\section{CONCLUSION AND DISCUSSION}

In this paper, based on the cause analysis on boundary artifacts in image deconvolution, we suggest not to use any specific $\mathrm{BCs}$ and to use the normalized backprojector for the boundary artifact removal. By not using any specific $\mathrm{BCs}$, the proposed method is immune to impose mismatched $\mathrm{BCs}$ on unseen pixels. By using the normalized backprojector, the proposed method can remove boundary artifacts effectively. Simulation results supported described claims by showing that the proposed method removes boundary artifacts more effectively than $\mathrm{PBC}, \mathrm{RBC}$, and $\mathrm{ABC}$, with only few extra computations than $\mathrm{PBC}$ based methods.

\section{REFERENCE}

[1] R. Gonzalez, and R. Woods. Digital Image Processing Prentice-Hall, Englewood Cliffs, NJ. 2002.

[2] S. Haykin. Adaptive Filtering Theory. Prentice-
Hall, Inc. Upper Saddle River, NJ, USA.1986.

[3] D. Calvetti, and E. Somersalo, "Bayesian Image Deblurring and Boundary Effects," Proceeding of Society of Photo-Optical Instrumentation Engineers (SPIE) Conference Series, Vol. 5910, pp. 281-289. 2005.

[4] W. H. Richardson, "Baysian-based Iterative Method of Image Restoration," Journal of the Optical Society of America, Vol. 62, No. 1, pp. 55-59, 1972.

[5] L. B. Lucy, "An Iterative Technique for The Rectification of Observed Distributions," The Astronomical Journal, Vol. 79, No. 6, pp. 745754, 1974.

[6] D. Donoho, "Nonlinear Solution of Linear Inverse Problems by Wavelet-vagulette Decomposition," Applied and Computational Harmonic Analysis, Vol. 2, No. 2, pp. 101-126, 1995.

[7] R. Chan, T. Chan, L. Shen, and Z. Shen, "Wavelet Deblurring Algorithms for Spatially Varying Blur from High-resolution Image Reconstruction," Linear Algebra and its Applications, Vol. 366, No. 1, pp.139-155, 2003.

[8] R. Rudin and S. Osher, "Total Variation based Image Restoration with Free Local Constraints," Proceeding of IEEE International Conference Image Processing, Vol. 1. pp. 31-35, 1994.

[9] M. Welk, D. Thesi, and J. Weickert, "Variational Deblurring of Images with Uncertain and Spatially Variant Blurs," Pattern Recognition, Vol. 3663, No. 1, pp. 485-492, 2005.

[10] S.A. Yoon, T.G. Lee, S.H Lee, M.K. Son, D.G. Kim and C.H. Won "Image Resolution Enhancement by Improved S\&A Method using POCS," Journal of Korea Multimedia Society, Vol. 14, No. 11, pp. 1392-1400, 2011.

[11] A. Tekalp and M. Sezan, "Quantitative Analysis of Artifacts in Linear Space-invariant Image Restoration," Multidimensional Systems and Signal Processing, Vol. 1, No. 2, pp. 143-177, 1990. 
[12] J. Nagy, K. Palmer, and L. Perrone, "Iterative Methods for Image Deblurring: A Matlab Oriented Approach," Numerical Algorithms, Vol. 36, No. 1, pp. 73-93, 2004.

[13] M. Ng, R. Chan, and W.-C. Tang, "A Fast Algorithm for Deblurring Models with Neumann Boundary Donditions," SIAM Journal on Scientific Computing, Vol. 21, No. 3, pp. 851-866, 1999.

[14] M. Hanke, J. Nagy, and R. Plemmons, "Preconditioned Iterative Regularization Methods for Ill-posed Problems," in Numerical Linear Algebra and Scientific Computing, eds. L. Reichel, pp. 141-163. de Gruyter, Berlin, Germany, 1993.

[15] S. Sera-Capizzano, "A Note on Anti-reflective Boundary Conditions and Fast Deblurring Models," SIAM Journal of Scientific Computing. Vol. 25, No. 4, 1307- 1325, 2003.

[16] D. Calvetti, J. Kaipio, and E. Someralo, "Aristotelian prior Boundary Conditions," International Journal of Mathematics and Computer Science, Vol. 1, No. 1, pp. 63-81, 2006.

[17] H. W. Engl, M. Hanke, and A. Neubauer, Regularization of Inverse Problems, Kluwer Academic Publishers, Dordrecht, 2000.

[18] M. R. Hestenes and E. Stiefel, "Methods of Conjugate Gradients for Solving Linear Systems," Journal of Research of the National Bureau of Standards, Vol. 49, No. 6, pp. 409436, 1952.

[19] M. Bertero and P. Boccacci, "A Simple Method for The Reduction of Boundary Effects in The Richardson-Lucy Approach to Image Deconvolution," Astronomy and Astrophysics, Vol. 437, No. 1, pp. 369-374, 2005.
[20] N.-Y. Lee and B. Lucier, Preconditioned Conjugate Gradient Method for Boundary Artifacts-free Image Deblurring, Technical Report 3-CNA-011, Center for Nonlinear Analysis, Carnegie Mellon University, 2013 (Submitted).

[21] G. L. Zeng and C. T. Gullberg, "Unmatched Projector/Backprojector Pairs in an Iterative Reconstruction Algorithm," IEEE Transaction on Medical Imaging, Vol. 19, No. 5, pp. 548555, 2000.

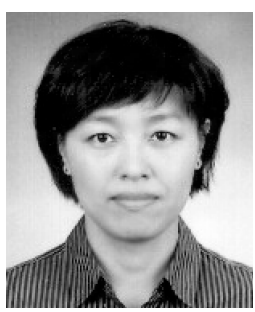

\section{Ji-Yeon Lee}

2011. 3 2014. 8 Inje University, Department of Computer Aided Science, Ph.D.

Research Iterests: Numerical Analysis based Image Processing

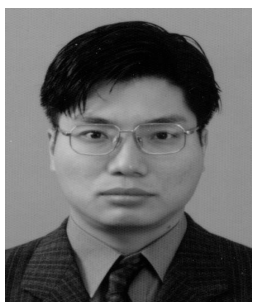

Nam-Yong Lee

1992. 9 1997 12 Purdue University, Ph.D.

2002. 9 Current Inje University, Department of Applied Mathematics, Associate Professorr

Research Interests: Image Restoration, Medical Imaging, Contents Protection 